\title{
Micromagnetic Simulation of Strain-Assisted Current-Induced Magnetization Switching
}

\author{
H. B. Huang, C. P. Zhao, and X. Q. Ma \\ Department of Physics, University of Science and Technology Beijing, Beijing 100083, China \\ Correspondence should be addressed to H. B. Huang; houbinghuang@gmail.com
}

Received 13 June 2016; Revised 30 July 2016; Accepted 2 August 2016

Academic Editor: Jingsheng Chen

Copyright ( $) 2016$ H. B. Huang et al. This is an open access article distributed under the Creative Commons Attribution License, which permits unrestricted use, distribution, and reproduction in any medium, provided the original work is properly cited.

\begin{abstract}
We investigated the effect of substrate misfit strain on the current-induced magnetization switching in magnetic tunnel junctions by combining micromagnetic simulation with phase-field microelasticity theory. Our results indicate that the positive substrate misfit strain can decrease the critical current density of magnetization switching by pushing the magnetization from out-of-plane to in-plane directions, while the negative strain pushes the magnetization back to the out-of-plane directions. The magnetic domain evolution is obtained to demonstrate the strain-assisted current-induced magnetization switching.
\end{abstract}

\section{Introduction}

Since Sun et al. [1] and Sun et al. [2] proposed the spin transfer torque (STT) effects in the ferromagnetic materials, STT has attracted great attention due to its potential applications of magnetic devices in the past decades [3-8]. In STT effects, angular momentums of the electrons may transfer to the ferromagnetic magnetization. There are three major effects in STT driven magnetization dynamics: (1) current-induced magnetization switching [9-12], (2) current-driven magnetization oscillations $[4,13,14]$, and (3) current-driven domain wall motion [15-20]. The current-induced magnetization switching is used in the application of magnetic random access memory (MRAM), which has the advantage of large storage density, high switching speed, low energy consumption, and avoidance of cross writing. The current-induced magnetization switching also eliminates crosstalk between neighboring cells during writing [21]. Furthermore, STTMRAM has practically unlimited endurance and requires less energy and is faster than conventional magnetic field control MRAM. However, the high critical switching current density $J_{c}$ of STT-MRAM has to be reduced to achieve the compatibility with the metal-oxide-semiconductor technology.

There are many attempts to reduce $J_{c}$ for magnetization switching, for example, using $\mathrm{CoFeB}$ as the free layer to reduce the saturation magnetization $M_{S}$ [22]; using a double spin-filter structure [23] and an antiferromagnetic pinning structure [24] or inserting a $\mathrm{Ru}$ spin scattering layer to increase spin scattering [25]; using a composite free layer consisting of two ferromagnetic layers with various coupling types [26-28]; or using Heusler-based spin valve nanopillar [29]. In magnetic thin films, the strain can be effectively utilized to tune the magnetic domain structures [30-33]. For example, the magnetization can be switched between an inplane and out-of-plane orientation under isotropic biaxial inplane strains $[34,35]$ or rotate within the film plane under anisotropic biaxial in-plane strains [36]. Recently, Pertsev and Kohlstedt [37] demonstrated that the critical current density needed for $180^{\circ}$ magnetization switching in the free magnetic layer of a spin valve can be reduced drastically by the assistance of substrate misfit strain based on the thermodynamic calculation. Thermodynamic calculation minimizes the total free energy to obtain homogeneous magnetization distribution, but micromagnetic simulations provide the microstructure evolution of magnetization switching. The conventional micromagnetic simulations do not take account of such elastic effect and thus cannot be employed to investigate the assistance of substrate misfit strain in STT induced magnetization switching.

In this paper, we combined the phase-field microelasticity theory with micromagnetic simulations to illustrate misfit 
strain which causes the decrease of the critical current density for magnetization switching. In particular, we investigated STT induced $180^{\circ}$ magnetization switching in CoFeB magnetic tunnel junctions based on the misfit strain induced $90^{\circ}$ magnetization switching [38]. Firstly, we discussed our results by using a hysteresis loop to show the decrease of critical current density. Then, we discussed the effect of substrate strain assistance in STT induced magnetization switching by showing magnetization and magnetic domain evolutions.

\section{Model Description}

As shown in Figure 1, we investigate the spin valve nanopillars with the structure of $\mathrm{CoFeB}(2 \mathrm{~nm}) / \mathrm{MgO}(2 \mathrm{~nm}) / \mathrm{CoFeB}$ $(16 \mathrm{~nm})$ of square cross-sectional area $\left(80 \times 80 \mathrm{~nm}^{2}\right)$. A Cartesian coordinate system is employed and the current is along the $z$-axis. A thin $\mathrm{MgO}$ layer separates the two $\mathrm{CoFeB}$ layers, and the bottom $\mathrm{CoFeB}$ layer is the free layer. A spin-polarized current drives the magnetization dynamics of free layer. The top CoFeB layer is the fixed layer with its magnetization vector $\mathbf{P}$ along the positive $z$-axis. The initial magnetization vector $\mathbf{M}$ of the layer is along the negative or positive $z$-axis. The lateral length of the free layer of spin valve is fully constrained by a stiff substrate. We generally define the substrate strain $\varepsilon_{i i}(i=1,2)$. The positive current is defined as electrons flowing from the fixed layer to the free layer. In the bottom, a ferroelectric (FE) layer is used to adjust the output strain. In this paper, the positive current will lead to the parallel structure (P, "1") while the negative current will lead to the antiparallel structure (AP, “0”) according to STT theory.

We use a generalized Landau-Lifshitz-Gilbert-Slonczewski (LLGS) equation to describe the magnetization dynamics $[39,40]$,

$$
\begin{aligned}
\frac{d \mathbf{M}}{d t}= & -\gamma^{\prime} \mathbf{M} \times \mathbf{H}_{\mathrm{eff}}-\frac{\alpha \gamma^{\prime}}{M_{s}} \mathbf{M} \times\left(\mathbf{M} \times \mathbf{H}_{\mathrm{eff}}\right) \\
& -\frac{2 \mu_{B} J}{\left(1+\alpha^{2}\right) e d M_{s}^{3}} g(\mathbf{M}, \mathbf{P}) \mathbf{M} \times(\mathbf{M} \times \mathbf{P}) \\
& +\frac{2 \mu_{B} \alpha J}{\left(1+\alpha^{2}\right) e d M_{s}^{2}} g(\mathbf{M}, \mathbf{P})(\mathbf{M} \times \mathbf{P}),
\end{aligned}
$$

where the first term represents the precession torque and the second term represents the Gilbert damping torque. $\mathbf{H}_{\mathrm{eff}}$ is the effective field, $\gamma^{\prime}=\gamma /\left(1+\alpha^{2}\right), \gamma$ is the electron gyromagnetic ratio, and $\alpha$ is the dimensionless damping parameter. The effective field includes the anisotropy field, the demagnetization field, the external field, the elastic field, and the exchange field; namely, $\mathbf{H}_{\mathrm{eff}}=\mathbf{H}_{k}+\mathbf{H}_{d}+\mathbf{H}_{\mathrm{ext}}+\mathbf{H}_{\mathrm{elas}}+\mathbf{H}_{\mathrm{ex}}$, given as

$$
\mathbf{H}_{\mathrm{eff}}=-\frac{1}{\mu_{0}} \frac{\delta E}{\delta \mathbf{M}}
$$

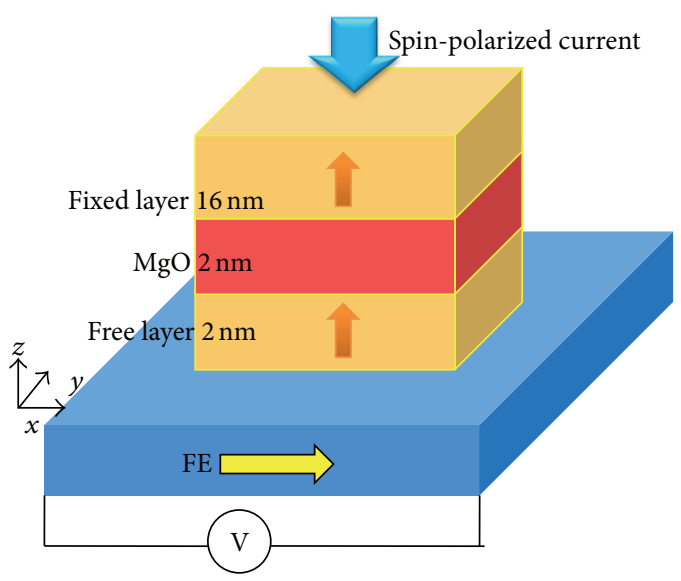

Figure 1: Schematic structure of CoFeB $(2 \mathrm{~nm}) / \mathrm{MgO}(2 \mathrm{~nm}) / \mathrm{CoFeB}$ $(16 \mathrm{~nm})$ magnetic tunnel junction spin valve.

where $E$ is the total energy, expressed by $E=E_{k}+E_{d}+$ $E_{\text {ext }}+E_{\text {elas }}+E_{\text {ex }}$, where $E_{k}, E_{d}, E_{\text {ext }}, E_{\text {elas }}$, and $E_{\text {ex }}$ are anisotropy energy (including magnetocrystalline and shape anisotropies) [41], demagnetization energy, Zeeman energy, elastic energy, and exchange energy, respectively. The details for obtaining $E_{k}, E_{d}, E_{\text {ext }}, E_{\text {ex }}$, and $E_{\text {elas }}$ can be found in our previous papers [41-43]. The last two terms on the right side of (1) describe STT that tends to drag the magnetization away from its initial state to its final state. The scalar function is given by $[39,40], g(\mathbf{M}, \mathbf{P})=\left[-4+(1+\eta)^{3}(3+\mathbf{M}\right.$. $\left.\left.\mathbf{P} / M_{s}^{2}\right) / 4 \eta^{3 / 2}\right]^{-1}$, where $\eta$ is the spin polarization constant, $\mathbf{M}$ and $\mathbf{P}$ are the magnetization of free and fixed layers in Figure 1, and the angle between $\mathbf{M}$ and $\mathbf{P}$ is $\theta \cdot \mathbf{M} \cdot \mathbf{P} / M_{s}^{2}=$ $\cos \theta . \mathbf{H}_{\text {STT }}$ is the corresponding effective field given by $\mathbf{H}_{\text {STT }}=2 \mu_{B} J g(\mathbf{M}, \mathbf{P}) \mathbf{M} \times \mathbf{P} /\left(\right.$ jed $\left.M_{s}^{3}\right)$, where $\mu_{B}, J, d, e$, and $M_{s}$ are the Bohr magneton, current density, thickness of the free layer, electron charge, and saturation magnetization, respectively.

The magnetic parameters employed in the simulations are as follows: saturation magnetization $M_{s}=9.549 \times 10^{5} \mathrm{~A} / \mathrm{m}$ [44, 45], Gilbert damping parameter $\alpha=0.00439$ [46], spin polarization factor $\eta=0.5$ [47], magnetic anisotropy constants $K_{1}=1.2 \times 10^{4} \mathrm{~J} / \mathrm{m}^{3}$ and $K_{2}=0$ [37], elastic constants $c_{11}=2.57 \times 10^{11} \mathrm{Nm}^{-2}, c_{12}=1.62 \times 10^{11} \mathrm{Nm}^{-2}$, and $c_{44}=1.05 \times 10^{11} \mathrm{Nm}^{-2}$ [37], and magnetostrictive constants $\lambda_{100}=139 \mathrm{ppm}$ and $\lambda_{111}=22 \mathrm{ppm}[44,45]$. We investigated the influence of normal substrate strains $\varepsilon_{11}$ and $\varepsilon_{22}$ on the magnetization state by assuming a zero shear strain. The dynamics of magnetization was investigated by numerically solving the time-dependent LLGS equation using the Gauss-Seidel projection method and the semiimplicit Fourier spectral method [48-53]. The samples were discretized in computational cells of $2 \times 2 \times 2 \mathrm{~nm}^{3}$.

\section{Results and Discussions}

Figure 2 shows the hysteresis loops with the substrate misfit strains $\left(\varepsilon_{11}=\varepsilon_{22}=0\right.$ and $\left.0.5 \%\right)$. Without the applied 


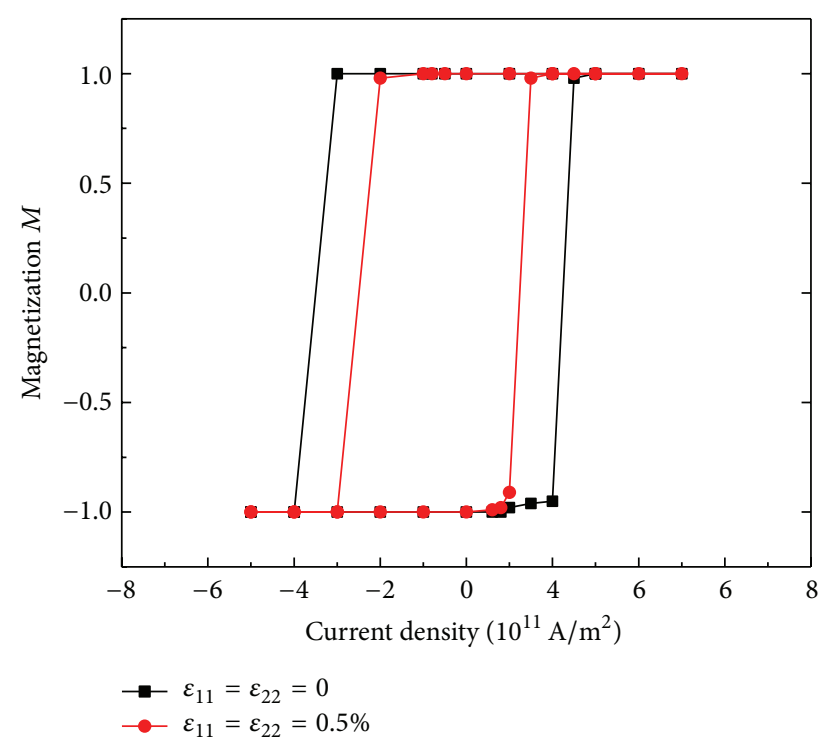

FIGURE 2: Hysteresis loops of magnetization versus current density with strain and without strain.

strain, there is a square hysteresis loop of magnetization versus current density $(M-J)$ curve. The critical current densities are $2.1 \times 10^{11} \mathrm{~A} / \mathrm{m}^{2}$ from AP to $\mathrm{P}$ and $-3.7 \times$ $10^{11} \mathrm{~A} / \mathrm{m}^{2}$ from $\mathrm{P}$ to AP. At the biaxial strain $0.5 \%$, we observe the decrease of critical current densities for magnetization switching (red hysteresis loop). The critical current densities are $1.3 \times 10^{11} \mathrm{~A} / \mathrm{m}^{2}$ from AP to $\mathrm{P}$ and $-2.7 \times$ $10^{11} \mathrm{~A} / \mathrm{m}^{2}$ from $\mathrm{P}$ to AP. The positive substrate misfit strain reduces the critical current densities by pushing the magnetization from the out-of-plane to the in-plane directions. Therefore, the substrate misfit strain can be used effectively in assisting the STT induced magnetization switching.

Figure 3 shows the evolution of magnetization components $\left\langle m_{z}\right\rangle$ with different current densities and substrate misfit strains. For the black line, the magnetization is along the initial $-z$-axis and keeps the AP structure without the applied current and strain. The red and pink lines represent the magnetization switching from $\mathrm{AP}$ to $\mathrm{P}$ and $\mathrm{P}$ to $\mathrm{AP}$ at the current densities of $1.0 \times 10^{12} \mathrm{~A} / \mathrm{m}^{2}$ and $-1.0 \times 10^{12} \mathrm{~A} / \mathrm{m}^{2}$. Since the applied current densities are large enough to overcome the barrier, the magnetization will switch from AP to $\mathrm{P}$ or $\mathrm{P}$ to $\mathrm{AP}$ at the negative or positive current densities. We take the magnetization switching from AP to P, for example, to show the effect of misfit strain. If the biaxial positive strain $\varepsilon_{11}=\varepsilon_{22}=0.5 \%$ is applied, the magnetization switching (cyan line) is faster than that without strain (red line). However, the biaxial negative strain $\varepsilon_{11}=\varepsilon_{22}=$ $-0.5 \%$ will prolong the time of the magnetization switching (blue line). The mechanism of the phenomenon resulted from the fact that the positive strain will drag the magnetization along the in-plane direction, while the negative strain will push the magnetization back to the out-of-plane direction.

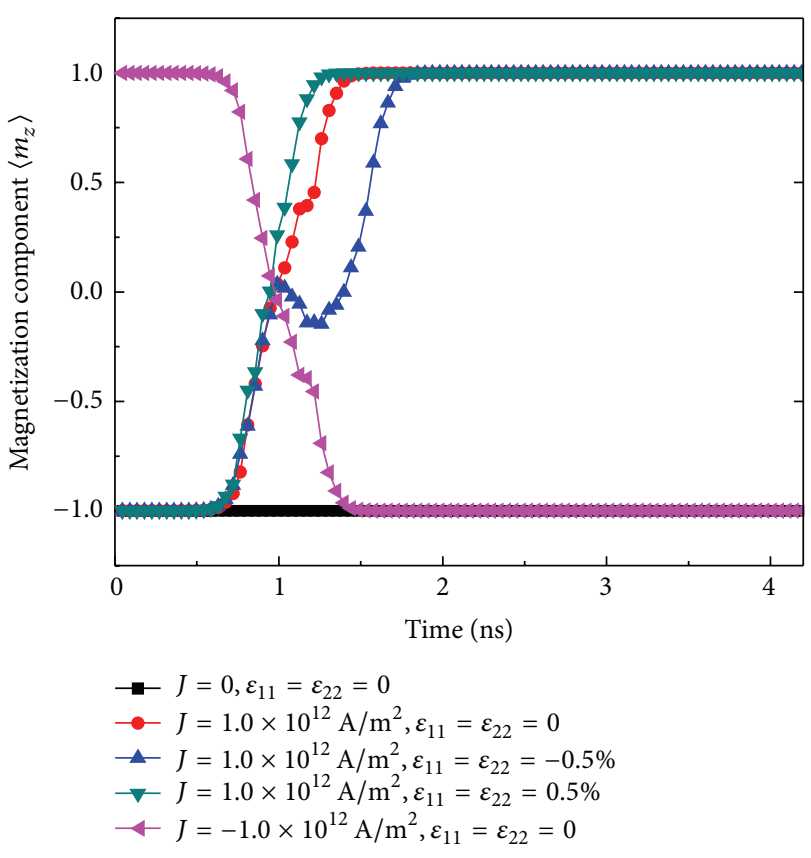

FIGURE 3: Time evolution of magnetization components $\left\langle m_{z}\right\rangle$ with different current densities and substrate misfit strains.

We can use the magnetic domain evolution to elucidate the effect of misfit strain. As shown in Figure 4, we show the corresponding magnetization distributions at the current density $1.0 \times 10^{12} \mathrm{~A} / \mathrm{m}^{2}$ under different applied strains. Three rows correspond to the magnetization switching of red (zero strain), cyan $(0.5 \%)$, and blue $(-0.5 \%)$ lines in Figure 3 . The colors represent different domain area: purple is $-z$, red is $+z$, and other colors are domain wall area. We observed that the magnetization was switched from the initial $-z$-axis to the final $+z$-axis due to the large current density input. This multidomain evolution process resulted from the large current input energy. In the first row, the initial magnetization was along $-z$-axis. Without the strain, the magnetization switching process started from the outer to the inner of the free layer. However, the magnetization switching process started from the inner to the outer of the free layer in the negative strain. For the positive strain, we can observe that the nucleation starts from the center and diffuses to the edges.

\section{Conclusions}

In conclusion, we investigated the strain-assisted STT induced magnetization switching in CoFeB-based magnetic tunnel junctions by combining phase-field method with micromagnetic simulations. We proposed an effective method of using strain-assisted STT induced magnetization switching instead of using an external magnetic field. Since the tensile biaxial substrate misfit strains drag the magnetization into the in-plane directions, the critical current density and switching time are decreased significantly. 


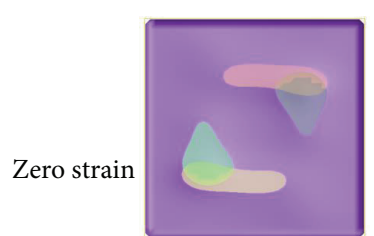

$0.5(\mathrm{~ns})$

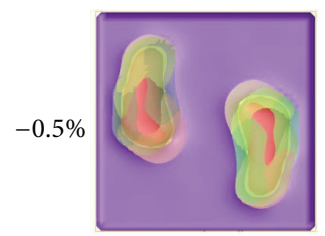

$0.5(\mathrm{~ns})$

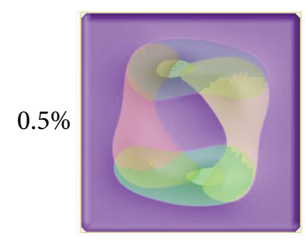

0.5 (ns)

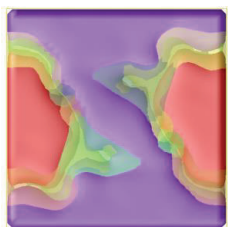

$1.0(\mathrm{~ns})$

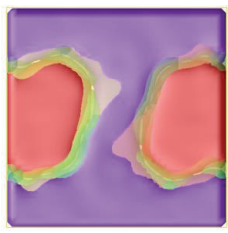

1.0 (ns)

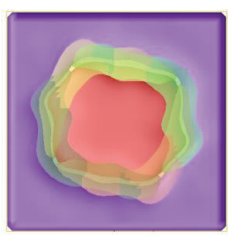

$1.0(\mathrm{~ns})$

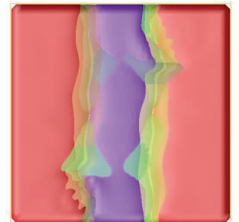

$1.2(\mathrm{~ns})$

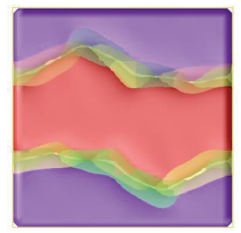

1.2 (ns)

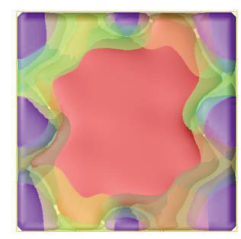

$1.2(\mathrm{~ns})$

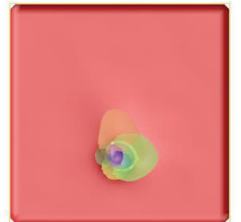

$1.5(\mathrm{~ns})$

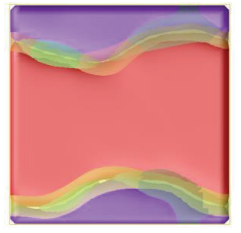

1.5 (ns)

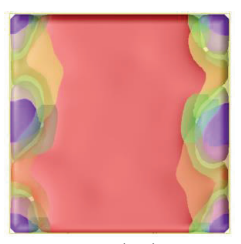

1.5 (ns)

FIGURE 4: Magnetic domain evolutions with different misfit substrate strains during the magnetization switching.

\section{Competing Interests}

The authors declare that there are no competing interests regarding the publication of this paper.

\section{Acknowledgments}

This work was sponsored by the National Science Foundation of China (11504020).

\section{References}

[1] J. Z. Sun, J. DeBrosse, and P. K. Wang, "Feasibility of an active-read approach for spin-torque switched magnetic tunnel junctions in a random access memory," Journal of Applied Physics, vol. 114, no. 9, Article ID 094503, 2013.

[2] G. Y. Sun, E. Kursun, J. A. Rivers, and Y. Xie, "Exploring the vulnerability of CMPs to soft errors with $3 \mathrm{D}$ stacked nonvolatile memory," ACM Journal on Emerging Technologies in Computing Systems, vol. 9, no. 3, article 22, 2013.

[3] J. A. Katine, F. J. Albert, R. A. Buhrman, E. B. Myers, and D. C. Ralph, "Current-driven magnetization reversal and spin-wave excitations in $\mathrm{Co} / \mathrm{Cu} / \mathrm{Co}$ pillars," Physical Review Letters, vol. 84, no. 14, pp. 3149-3152, 2000.

[4] S. Kaka, M. R. Pufall, W. H. Rippard, T. J. Silva, S. E. Russek, and J. A. Katine, "Mutual phase-locking of microwave spin torque nano-oscillators," Nature, vol. 437, no. 7057, pp. 389-392, 2005.

[5] S. I. Klselev, J. C. Sankey, I. N. Krivorotov et al., "Microwave oscillations of a nanomagnet driven by a spin-polarized current," Nature, vol. 425, no. 6956, pp. 380-383, 2003.

[6] I. Lee, Y. Obukhov, G. Xiang et al., "Nanoscale scanning probe ferromagnetic resonance imaging using localized modes," Nature, vol. 466, no. 7308, pp. 845-848, 2010.

[7] M. Tsol, A. G. M. Jansen, J. Bass, W.-C. Chlang, V. Tsol, and P. Wyder, "Generation and detection of phase-coherent currentdriven magnons in magnetic multilayers," Nature, vol. 406, no. 6791, pp. 46-48, 2000.
[8] D. Ralph and M. Stiles, "Spin transfer torques," Journal of Magnetism and Magnetic Materials, vol. 320, no. 7, pp. 1190-1216, 2008.

[9] E. B. Myers, D. C. Ralph, J. A. Katine, R. N. Louie, and R. A. Buhrman, "Current-induced switching of domains in magnetic multilayer devices," Science, vol. 285, no. 5429, pp. 867-870, 1999.

[10] J. Grollier, P. Boulenc, V. Cros et al., "Switching a spin valve back and forth by current-induced domain wall motion," Applied Physics Letters, vol. 83, no. 3, pp. 509-511, 2003.

[11] Y. Liu, Z. Zhang, P. P. Freitas, and J. L. Martins, "Currentinduced magnetization switching in magnetic tunnel junctions," Applied Physics Letters, vol. 82, no. 17, pp. 2871-2873, 2003.

[12] B. Özyilmaz, A. D. Kent, D. Monsma, J. Z. Sun, M. J. Rooks, and R. H. Koch, "Current-induced magnetization reversal in high magnetic fields in Co/Cu/Co nanopillars," Physical Review Letters, vol. 91, no. 6, Article ID 067203, 2003.

[13] D. V. Berkov, "Micromagnetic simulations of the magnetization dynamics in nanostructures with special applications to spin injection," Journal of Magnetism and Magnetic Materials, vol. 300, no. 1, pp. 159-163, 2006.

[14] V. S. Pribiag, I. N. Krivorotov, G. D. Fuchs et al., "Magnetic vortex oscillator driven by d.c. spin-polarized current," Nature Physics, vol. 3, no. 7, pp. 498-503, 2007.

[15] Z. Li and S. Zhang, "Domain-wall dynamics driven by adiabatic spin-transfer torques," Physical Review B, vol. 70, no. 2, Article ID 024417, 10 pages, 2004.

[16] G. Meier, M. Bolte, U. Merkt, B. Krüger, and D. Pfannkuche, "Current-induced domain-wall motion in permalloy semi rings," Journal of Magnetism and Magnetic Materials, vol. 316, no. 2, pp. e966-e968, 2007.

[17] C. Nam, Y. M. Jang, K. S. Lee, S. K. Lee, T. W. Kim, and B. K. Cho, "Current-supported domain wall movement to the target spot with a magnetic field," Journal of Magnetism and Magnetic Materials, vol. 310, no. 2, pp. 2023-2025, 2007. 
[18] M. D. Stiles, W. M. Saslow, M. J. Donahue, and A. Zangwill, "Adiabatic domain wall motion and Landau-Lifshitz damping," Physical Review B, vol. 75, no. 21, Article ID 214423, 2007.

[19] G. S. D. Beach, M. Tsoi, and J. L. Erskine, "Current-induced domain wall motion," Journal of Magnetism and Magnetic Materials, vol. 320, no. 7, pp. 1272-1281, 2008.

[20] R. A. Duine, "Spin pumping by a field-driven domain wall," Physical Review B, vol. 77, no. 1, Article ID 014409, 2008.

[21] K. MacHida, N. Funabashi, K.-I. Aoshima et al., "Spin transfer switching of closely arranged multiple pillars with currentperpendicular-to-plane spin valves," Journal of Applied Physics, vol. 103, no. 7, Article ID 07A713, 2008.

[22] K. Yagami, A. A. Tulapurkar, A. Fukushima, and Y. Suzuki, "Low-current spin-transfer switching and its thermal durability in a low-saturation-magnetization nanomagnet," Applied Physics Letters, vol. 85, no. 23, pp. 5634-5636, 2004.

[23] G. D. Fuchs, J. A. Katine, S. I. Kiselev et al., "Spin torque, tunnel-current spin polarization, and magnetoresistance in MgO magnetic tunnel junctions," Physical Review Letters, vol. 96, no. 18, Article ID 186603, 2006.

[24] K. J. Lee, T. H. Y. Nguyen, and K.-H. Shin, "Reduction in critical current density of current-induced magnetization switching," Journal of Magnetism and Magnetic Materials, vol. 304, no. 1, pp. 102-105, 2006.

[25] Y. Jiang, G. H. Yu, Y. B. Wang et al., "Spin transfer in antisymmetric exchange-biased spin-valves," Applied Physics Letters, vol. 86, no. 19, Article ID 192515, pp. 1-3, 2005.

[26] H. Meng, J. Wang, and J.-P. Wang, "Low critical current for spin transfer in magnetic tunnel junctions," Applied Physics Letters, vol. 88, no. 8, Article ID 082504, 2006.

[27] Z. Zhang, Y. Zhang, Y. Liu, B. Ma, and Q. Y. Jin, "Spin-transferinduced magnetization switching in tunnel junctions with nanocurrent channels," Applied Physics Letters, vol. 90, no. 11, Article ID 112504, 2007.

[28] C.-T. Yen, W.-C. Chen, D.-Y. Wang et al., "Reduction in critical current density for spin torque transfer switching with composite free layer," Applied Physics Letters, vol. 93, no. 9, Article ID 092504, 2008.

[29] K. Aoshima, N. Funabashi, K. Machida, Y. Miyamoto, K. Kuga, and N. Kawamura, "Current induced magnetization reversal in spin valves with Heusler alloys," Journal of Magnetism and Magnetic Materials, vol. 310, no. 2, pp. 2018-2019, 2007.

[30] M. T. Johnson, P. J. H. Bloemen, F. J. A. Den Broeder, and J. J. De Vries, "Magnetic anisotropy in metallic multilayers," Reports on Progress in Physics, vol. 59, no. 11, pp. 1409-1458, 1996.

[31] D. Sander, "The correlation between mechanical stress and magnetic anisotropy in ultrathin films," Reports on Progress in Physics, vol. 62, no. 5, pp. 809-858, 1999.

[32] K. H. Ahn, T. Lookman, and A. R. Bishop, "Strain-induced metal-insulator phase coexistence in perovskite manganites," Nature, vol. 428, no. 6981, pp. 401-404, 2004.

[33] A. Mukherjee, W. S. Cole, P. Woodward, M. Randeria, and N. Trivedi, "Theory of strain-controlled magnetotransport and stabilization of the ferromagnetic insulating phase in manganite thin films," Physical Review Letters, vol. 110, no. 15, Article ID 157201, 2013.

[34] N. A. Pertsev, "Giant magnetoelectric effect via strain-induced spin reorientation transitions in ferromagnetic films," Physical Review B, vol. 78, no. 21, Article ID 212102, 2008.

[35] J. Wang, J.-M. Hu, L.-Q. Chen, and C.-W. Nan, "Strain-domain structure and stability diagrams for single-domain magnetic thin films," Applied Physics Letters, vol. 103, Article ID 142413, 2013.

[36] J.-M. Hu and C. W. Nan, "Electric-field-induced magnetic easy-axis reorientation in ferromagnetic/ferroelectric layered heterostructures," Physical Review B, vol. 80, no. 22, Article ID 224416, 2009.

[37] N. A. Pertsev and H. Kohlstedt, "Magnetoresistive memory with ultralow critical current for magnetization switching," Advanced Functional Materials, vol. 22, no. 22, pp. 4696-4703, 2012.

[38] J.-M. Hu, G. Sheng, J. X. Zhang, C. W. Nan, and L. Q. Chen, "Phase-field simulation of strain-induced domain switching in magnetic thin films," Applied Physics Letters, vol. 98, no. 11, Article ID 112505, 2011.

[39] L. Berger, "Emission of spin waves by a magnetic multilayer traversed by a current," Physical Review B, vol. 54, no. 13, pp. 9353-9358, 1996.

[40] J. C. Slonczewski, "Current-driven excitation of magnetic multilayers," Journal of Magnetism and Magnetic Materials, vol. 159, no. 1-2, pp. L1-L7, 1996.

[41] J.-M. Hu, Z. Li, J. Wang, and C. W. Nan, "Electric-field control of strain-mediated magnetoelectric random access memory," Journal of Applied Physics, vol. 107, no. 9, Article ID 093912, 2010.

[42] H. B. Huang, J. M. Hu, T. N. Yang, X. Q. Ma, and L. Q. Chen, "Strain-assisted current-induced magnetization reversal in magnetic tunnel junctions: a micromagnetic study with phase-field microelasticity," Applied Physics Letters, vol. 105, no. 12, Article ID 122407, 2014.

[43] K. M. D. Hals and A. Brataas, "Phenomenology of currentinduced spin-orbit torques," Physical Review B, vol. 88, no. 8, Article ID 085423, 2013.

[44] S. Ikeda, K. Miura, H. Yamamoto et al., "A perpendicularanisotropy $\mathrm{CoFeB}-\mathrm{MgO}$ magnetic tunnel junction," Nature Materials, vol. 9, no. 9, pp. 721-724, 2010.

[45] S. Yan, Z. L. Sun, and Y. B. Bazaliy, "Modification of the Stoner-Wohlfarth astroid by a spin-polarized current: an exact solution," Physical Review B, vol. 88, no. 5, Article ID 054408, 2013.

[46] C. A. F. Vaz, J. A. C. Bland, and G. Lauhoff, "Magnetism in ultrathin film structures," Reports on Progress in Physics, vol. 71, no. 5, Article ID 056501, 2008.

[47] J. P. Cascales, D. Herranz, J. L. Sambricio, U. Ebels, J. A. Katine, and F. G. Aliev, "Magnetization reversal in sub-100 nm magnetic tunnel junctions with ultrathin $\mathrm{MgO}$ barrier biased along the hard axis," Applied Physics Letters, vol. 102, no. 9, Article ID 092404, 2013.

[48] Y. Kajiwara, K. Uchida, D. Kikuchi, T. An, Y. Fujikawa, and E. Saitoh, "Spin-relaxation modulation and spin-pumping control by transverse spin-wave spin current in $\mathrm{Y}_{3} \mathrm{Fe}_{5} \mathrm{O}_{12}$," Applied Physics Letters, vol. 103, no. 5, Article ID 052404, 2013.

[49] H. B. Huang, X. Q. Ma, Z. H. Liu, C. P. Zhao, and L. Q. Chen, "Modelling current-induced magnetization switching in Heusler alloy Co 2FeAl-based spin-valve nanopillar," Journal of Applied Physics, vol. 115, no. 13, Article ID 133905, 2014.

[50] H. B. Huang, X. Q. Ma, Z. H. Liu, C. P. Zhao, S. Q. Shi, and L. Q. Chen, "Simulation of multilevel cell spin transfer switching in a full-Heusler alloy spin-valve nanopillar," Applied Physics Letters, vol. 102, no. 4, Article ID 042405, 2013.

[51] H. B. Huang, X. Q. Ma, Z. H. Liu, and L. Q. Chen, "Modelling high-power spin-torque oscillator with perpendicular magnetization in half-metallic Heusler alloy spin valve nanopillar," Journal of Alloys and Compounds, vol. 597, pp. 230-235, 2014. 
[52] H. B. Huang, X. Q. Ma, Z. H. Liu et al., "Micromagnetic simulation of spin-transfer switching in a full-Heusler $\mathrm{Co}_{2} \mathrm{FeAl}_{0.5} \mathrm{Si}_{0.5}$ alloy spin-valve nanopillar," Journal of Applied Physics, vol. 110, no. 3, Article ID 033913, 2011.

[53] H. B. Huang, X. Q. Ma, C. P. Zhao, Z. H. Liu, and L. Q. Chen, "Micromagnetic study of high-power spin-torque oscillator with perpendicular magnetization in half-metallic Heusler alloy spin valve nanopillar under external magnetic fields," Journal of Magnetism and Magnetic Materials, vol. 373, pp. 10-15, 2015. 

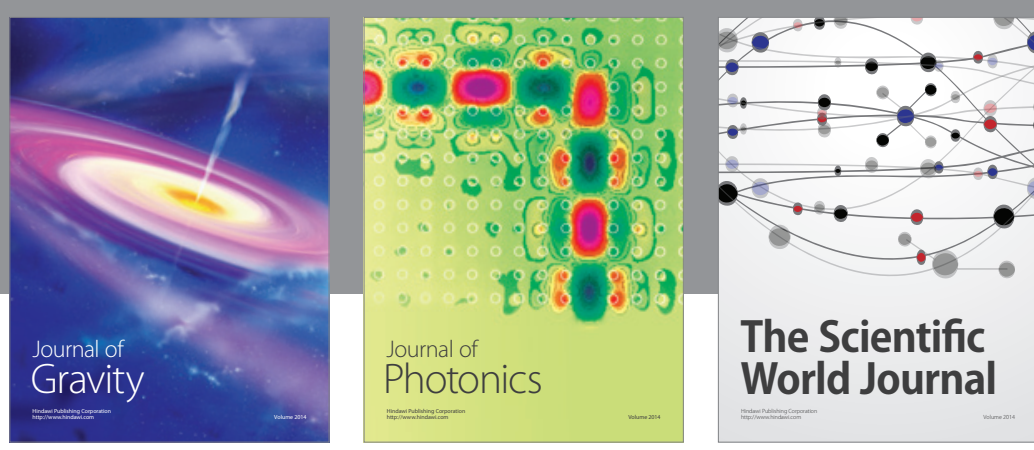

The Scientific World Journal
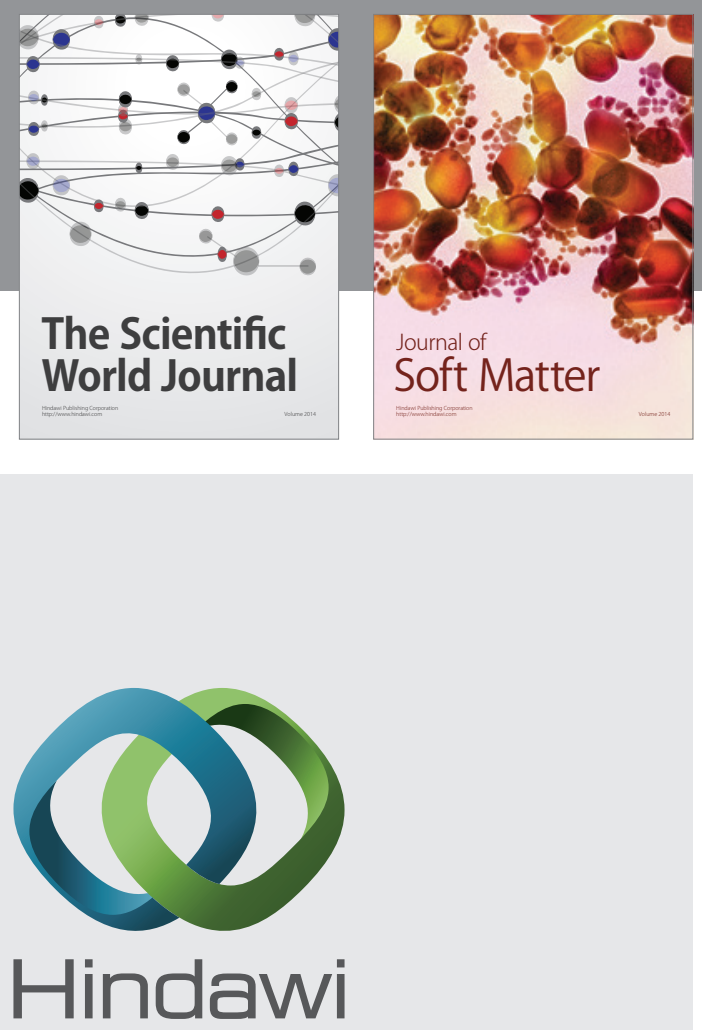

Submit your manuscripts at

http://www.hindawi.com

nternational Journal of

Statistical Mechanics
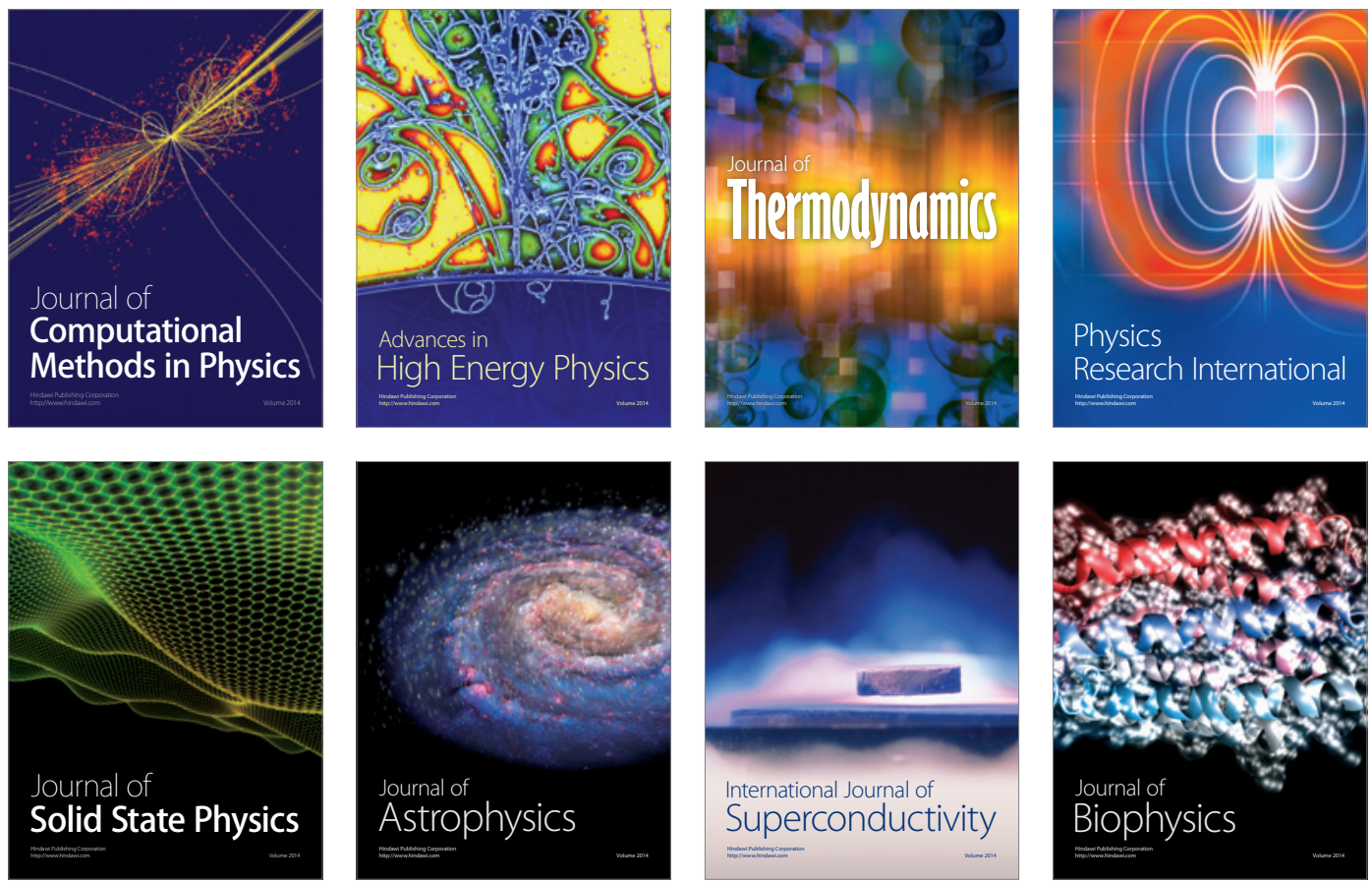
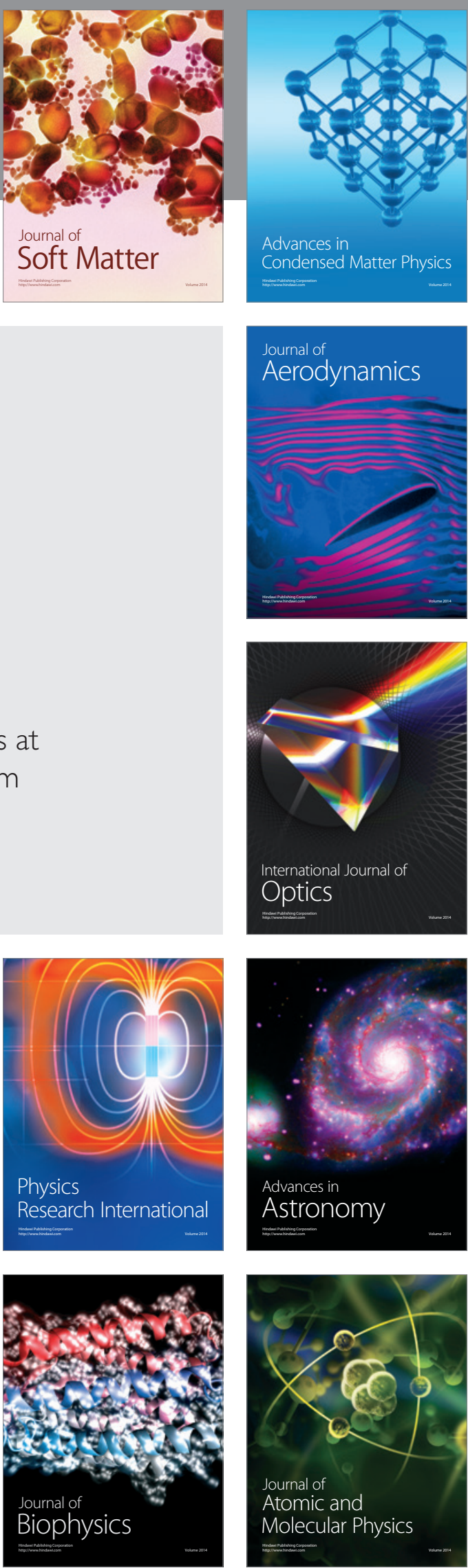